\title{
Management of Delay Optimization at Intersections
}

\author{
Łukasz Kuźmiński ${ }^{1}$, Piotr Maśloch ${ }^{2}{ }^{\circledR}$, Marek Bazan ${ }^{3}{ }^{\circledR}$, Tomasz Janiczek ${ }^{4}$,

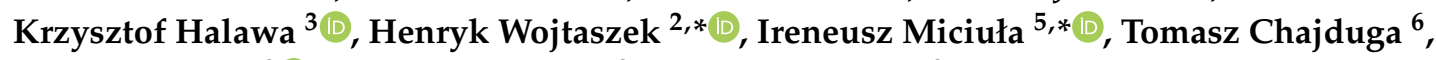 \\ Adam Kawecki ${ }^{3}$ D, Robert Czwartosz ${ }^{3}$ and Jakub Stawik ${ }^{3}$ \\ 1 Department of Process Management, Management Department, Wroclaw University of Economics, \\ 53-345 Wroclaw, Poland; lukasz.kuzminski@ue.wroc.pl \\ 2 Management Institute Management and Command Department, War Studies University, \\ 00-910 Warsaw, Poland; p.masloch@akademia.mil.pl \\ 3 Department of Computer Engineering, Faculty of Electronics, Wroclaw University of Science and \\ Technology, 50-372 Wrocław, Poland; marek.bazan@pwr.edu.pl (M.B.); krzysztof.halawa@pwr.edu.pl (K.H.); \\ 234944@student.pwr.edu.pl (A.K.); 226296@student.pwr.edu.pl (R.C.); 218715@student.pwr.edu.pl (J.S.) \\ 4 Department of Control Systems and Mechatronics, Faculty of Electronics, Wroclaw University of Science and \\ Technology, 50-372 Wroclaw, Poland; tomasz.janiczek@pwr.edu.pl \\ 5 Department of Sustainable Finance and Capital Markets, Faculty of Economics, Finance and Management, \\ University of Szczecin, 70-453 Szczecin, Poland \\ 6 Department of Logistics and International Management, Faculty of Management, Czestochowa University of \\ Technology, 42-218 Czestochowa, Poland; tomasz.chajduga@pcz.pl \\ * Correspondence: h.wojtaszek@akademia.mil.pl (H.W.); ireneusz.miciula@usz.edu.pl (I.M.)
}

Received: 14 May 2020; Accepted: 10 June 2020; Published: 15 June 2020

\begin{abstract}
Congestion extends the time of the journey for both people and goods. Therefore, transport solutions should be optimized. Management scientists and technical scientists worked together in order to develop a proprietary solution to increase efficiency in terms of productivity improvements for intelligent transport systems. The most fundamental functions of management have been paired with a detailed analysis of city traffic. The authors developed a method for determining the order of vehicles at traffic lights and connected it with vehicle-to-vehicle communication and GPS signals. As a result, a novel method to increase the throughput of intersections is presented. This solution generates a sound signal in order to inform the driver that the preceding car has started moving forward. The proposed solution leads to the shortening of the reaction time of the drivers waiting in a queue. This situation is most common at red lights. Consequently, the traffic simulation shows that the discharge of queues at traffic lights may be quicker by up to $13.5 \%$. Notably, that proposed solution does not require any modification of the infrastructure as well as any additional devices for vehicle-to-infrastructure communication at the road intersections. To conclude, proper implementation of the proposed solution will certainly contribute to efficiency improvements within intelligent transport systems, with the potential to reduce traffic jams.
\end{abstract}

Keywords: management; planning; organizing; motivating; controlling; vehicular ad-hoc networks; vehicle-to-vehicle data exchange; traffic lights; traffic congestion; driver reaction time; SUMO; OMNeT++

\section{Introduction}

Nowadays, due to the high transport load, there are very many cars on the streets, which significantly extend the time of journey for both residents and cargo transport. Therefore, the optimization of vehicles' movement in a traffic light queue is a subject of interest in the actual context of city traffic jams.

The authors agree that it is important to indicate the possibility of shortening the time of cars being present (moving and waiting) on the streets. This would free up space for other cars and increase 
the traffic flow. It is important to emphasize the aspect of the management of the traffic in the city. The most fundamental management functions, planning, organizing, motivating and controlling, can be developed in relation to city traffic, which definitely should be managed better in order to make it flow with more fluidity. The most fundamental management functions: planning, organizing, motivating and controlling, can be developed in relation to city traffic, which definitely should be managed better in order to make traffic more fluent.

With regard to technical aspects, the authors indicate that increasing road traffic flow while maintaining or improving road safety are the main objectives of all intelligent transport systems. Improving the infrastructure as well as increasing the capacity of roads are the most expensive parts of this task and all methods that enable the improvement of traffic flow with lower costs are especially desirable. From the point of view of the road user, there is the need for a reduction in travel time. Drivers vary in their behavior when they are waiting for a green light. The ideal situation would be that everyone was looking directly at the lights and starting to drive exactly at the moment when the green light comes on. However, this is not happening in real life. Many drivers ponder or do not focus on traffic lights when waiting for them to turn green. According to the literature, there are many reasons for drivers being distracted at traffic lights and not focused on observing if the vehicle in front of them has started to move forward [1]. Some drivers use their smartphones or change the settings of the navigation, radio, air conditioning and other devices whilst waiting at the lights. Parents can pay attention to children sitting in the rear of the vehicle. Sometimes people who get distracted and do not move from the lights for a long time, thereby blocking the traffic, are reminded of the necessity to move by the horns of vehicles behind. In [2], visual reaction times (VRT) and auditory reaction times (ART) were analyzed. It was found that ART is shorter than VRT, and that men react slightly faster than women to ART and VRT. In [3], the results of a study of response times of medical students to VRT visual stimuli and ART audio stimuli were described. For both women and men, response times to audio stimuli were significantly lower $(p<0.001)$ compared to visual stimuli. A large difference was found between male and female first-year students of medicine $(p<0.001)$, as well as between physically active and inactive students. The article written by Kacker, Saboo, Sharma and Nirvan [4] contains studies on the influence of various distracting factors in relation to ART and VRT. Moreover, it was found that the reaction times to ART audio stimuli were much shorter than VRT visual stimuli.

In this study, the idea is to install an intelligent notification system in vehicles which would make an audible sound when the preceding vehicle starts to move after the light turns green. As the results from the literature show, a sound signal indeed improves, i.e., shortens, the reaction time of the driver [2-4]. This would inform drivers waiting in a queue and force them to react faster when vehicle in the front of them starts moving. It is proposed that the moving vehicle will send a message to the next vehicle using Vehicular Ad-hoc Networks (VANETs) [5-9].

The main reasons of using VANET are as follows: to organize traffic during unpredictable situations-for example, an accident-to inform drivers of traffic congestion detection [10-13] or about the weather, to control the intersection [14-16], for accident avoidance and emergency management, cf. [17-19], and to enable the fastest path planning $[9,20]$. In all of the abovementioned aspects, using VANET leads to traffic jam reduction [21].

Let us underline here that the implementation of the presented method requires only a mobile application within each vehicle to be run while waiting at the lights, or a small device enabling accurate GNSS location readouts, VANET communication and buzzer functionality. None of the application connections to the engine or other electronic devices of vehicles are required, since moving off is fully controlled by the driver and therefore is no increased danger of an accident. The driver of the first vehicle reacts to the green light, whereas successive drivers react to the movement of the preceding vehicle. For all drivers, the reaction time is shortened by using the sound signal of the buzzer. The solution is safe and relatively inexpensive.

Finally, the proposed system may also be coupled with road safety applications using VANET communication. The GNSS device used to calculate vehicles' order and the buzzer functionality may 
be used as components of the various anti-crash systems that are able to handle such situations as emergency stops, blind spot cautioning, lane changing warnings and forward collision warnings [22].

To confirm the impact of the shortened reaction time of the drivers on the increase in the fluidity of traffic, the authors used such simulators as Simulation of Urban MObility (SUMO) [23], Veins and Objective Modular Network Tested in $\mathrm{C}++(\mathrm{OMNeT}++)$ [24]. Our original achievement is the presentation of a simulation for discharging a queue of vehicles at a single carriageway intersection with a vehicle column that can only go forward. The remainder of the paper is organized as follows. In Section 2, the proposed method for determining the order of vehicles at traffic lights using appropriate sequences of information exchange via VANET is described. The potential problems are mentioned and the operation of the method when some of the vehicles are not equipped with VANET is explained. Section 3.2 presents the simulation scenarios and results of the impact of shortening the drivers' reaction times to the movement of the preceding vehicle in relation to the flow of traffic at intersections with traffic lights. Finally, conclusions are offered at the end of the paper.

In order to improve the reaction time of drivers in a queue at red lights, the authors propose an original, user friendly notification system, based on a sound signal. As well offering a simple user experience, vehicle-to-vehicle communications and GNSS positioning are also used.

\section{Materials and Methods}

2.1. The Comparative Method-Core Management Functions (Methods) Analysed with Respect to the Proposed Solution and Aspect of City Traffic Management

One of the most common management functions (methods) characterized [25] are planning, organizing, motivation (leading) and controlling. During the development process, there were changes in terms of definitions and improvements. The names of these functions have changed, with different roles for employees and managers at different levels. Please see Figure 1 below.

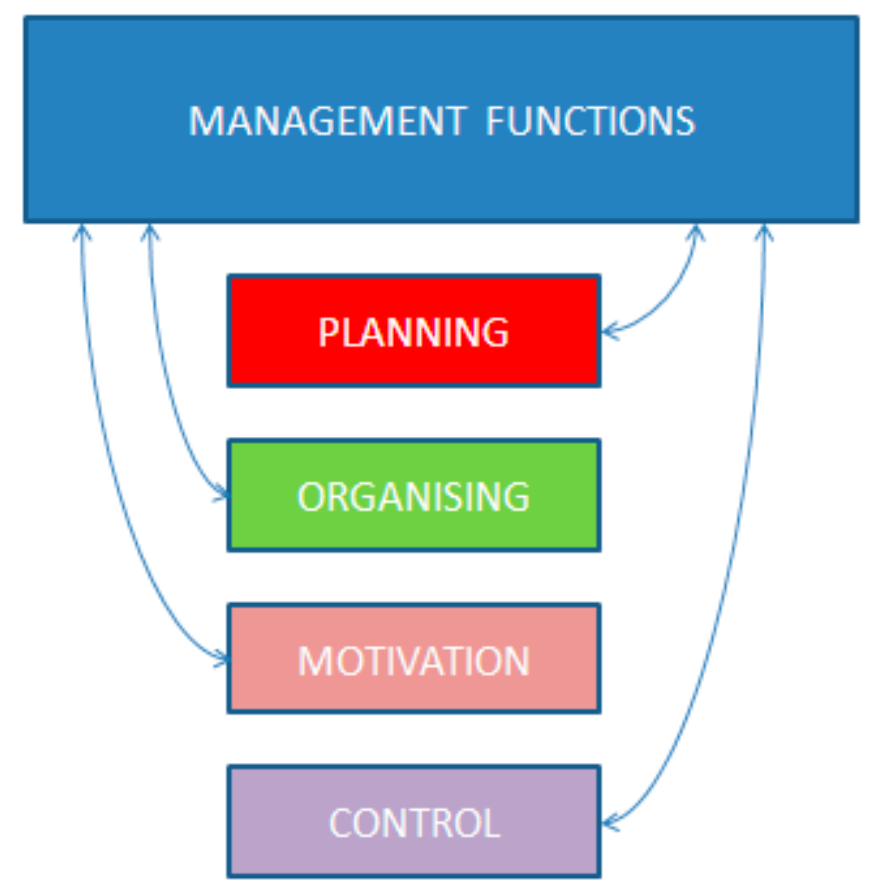

Figure 1. Basic management functions.

Planning is the fundamental first step to taking any action in managing an organization, team or project. Within the framework of planning, it is necessary to recognize and understand what exactly needs to be created in order to plan, predict future possible changes to the situation and determine the goals to be achieved. The necessary resources (technical, human, financial, etc.) should 
also be identified. One must also formulate the actions necessary to achieve the objectives, anticipate the consequences of taking or not taking action, and determine how to monitor and control the implementation of the plans [26].

Planning in the context of city traffic management may take the form of prediction, where (on which intersections) additional solutions are required in order to assure traffic flow in the city. This may take many more forms and be realized in many ways. For example, traffic lights can be programmed in such a way that the green light is no longer lit in directions where stronger traffic intensity is observed. The reason for this is to avoid causing traffic jams. With regard to the authors' proposed solution, it is worth mentioning that each town or city anticipating an increase in the number of cars, as well as traffic jams, should plan for the use of the proposed solution. The more cars that generally are in city traffic, the more time that is lost while waiting for the preceding drivers to start to drive after each light change. Therefore, planning to use the proposed solution would be useful in the fight against unnecessary traffic delays.

Organizing is about developing an appropriate resource mix to enable planned activities to be carried out properly and smoothly. Organization includes, among other things, spatial organization-e.g., the optimal placement of machines and equipment, division of work-distribution of tasks in a way that will ensure the full use of employees, without stoppages, but also without overload, and the development of the organizational structure-the organizational structure binds individual work posts into organizational units [26].

In terms of the management of traffic, organizing is very important in special situations. Such situations can be car accidents or any other event or situation resulting in different than usual traffic in specified places and at specific times. For example, this means that if a GPS recognizes that more cars than usual are standing still, it can advise a detour to others. Moreover, the traffic must be to some extent reorganized if, for example, ambulances take priority. The proposed solution easily supports the proper and expected reactions of drivers via early notification of the preceding car moving forward. The right behavior, it must be noted, requires a proper assessment of the situation by the driver.

All in all, because an efficient communication and information transfer system is important for the proper functioning of managerial areas $[27,28]$, this truth clearly also finds its application in the management of city traffic.

The motivation of this study is to modify the attitudes of people in such a way that they achieve more and act more effectively. It is believed that good decision making is the effect of, among other things, formal authority and professional competence [29]. The proposed solution is capable of giving solid information about the movement of the car in front. That is why, with time, it is expected to be treated as a professional tool to inform drivers about the movement of preceding cars and, in a way, to build some authority in this context, enabling faster reactions and reducing the waiting time. It is expected that sound informing the driver about the movement of preceding cars can be a kind of motivator to the act of releasing the brake and driving forward (stopping the driver from focusing on things other than driving). After all, if the horn signal from behind can be understood as a motivator, the signal from the onboard traffic system surely also can. Such behavior can also find its substantiation in the well-known Pavlov's dogs experiment from 1904.

Control involves checking whether the objectives set by the management in the planning process have been achieved. Thanks to control, it is possible to identify errors and indicate possibilities for the better performance of tasks in the future. In terms of the proposed solution, it does not fulfill the aspect of control as a function of management. However, it can be said that the driver, who rapidly receives information to show that they can start driving, also has better control over the vehicle. Anthony said, in 2007 [30], that the objectives of action should be reflected in concrete and measurable indicators. Here, the objective would be a smooth ride to the aim, while the indicator is, obviously, a sound signal generated by the proposed solution. 
Figure 2 shows the management implementation model, which was developed in order to implement the proposed solution at the highest possible level.

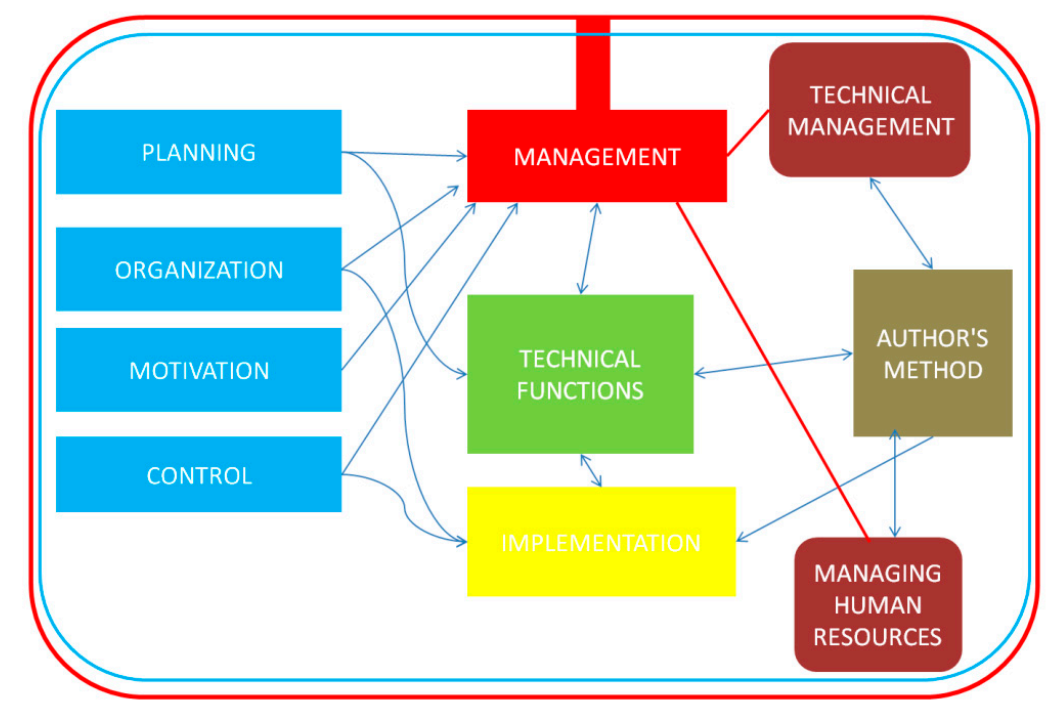

Figure 2. Management implementation model in combination with technical functions. Source: authors' visualization of their solution (method) among aspects of management (based on Hashiba and Chang, 1992).

The above model presents the essence of management or, more precisely, the connection of its basic management functions, such as planning, organizing, motivation and control with respect to technical aspects and implementation. The whole process has contributed to this work in the form of an original model [31] and would not be possible without the involvement of scientists from various interdisciplinary scientific disciplines [32-34].

\subsection{Proposed Method of Communication V2V at Traffic Lights Using VANETs}

This section describes the method for information exchange between vehicles located near traffic lights.

Here, the authors present an original application of VANETs to increase the fluidity of a sequence of vehicles starting to move forward at an intersection with traffic lights. VANETs are used to exchange geolocation data among neighboring vehicles so that calculations of the position of a vehicle versus other vehicles approaching the intersection are possible [35]. Geolocation with less than half a meter of accuracy is necessary in order to provide the correct order of vehicles broadcasting and receiving a signal. Once the geolocation of the preceding vehicle is obtained in two successive timestamps, one vehicle may establish whether another (the preceding) vehicle has already started to move. When the forward movement of the preceding vehicle is detected, the intelligent sound signal in the vehicle is activated to shorten the reaction time of the driver. A greater fluidity in the column of vehicles as they start to move at the traffic lights is achieved. The assumption is that the reaction time of each individual driver is shortened and that more time is saved in total. The empirical measurements of a safe headway are reported, e.g., in [36] and range safely from $1 \mathrm{~s}$ to $3 \mathrm{~s}$.

Moreover, the original results of measurements are presented. The measurements were carried out at a real intersection to calculate a headway between vehicles that start moving at a green light. One measurement was conducted via publicly available monitoring in Warsaw (Poland). The authors also present the measurements of pure reaction time upon the appearance of a green light at a set of traffic lights. The measurements were conducted on a sample group of 130 drivers using a web application that visualized the preceding vehicle stating to move randomly with and without a sound signal. Empirical distributions from the abovementioned measurements, i.e., reaction times upon the appearance of a 
green light with and without a sound signal are presented. The combined results allowed the authors to build a distribution of shortened headways between vehicles if the sound signal was emitted by the application connected via VANET when the preceding vehicle started to move.

The method for determining the order of vehicles is shown using several vehicles marked with letters A, B, and C (cf. Figures 3 and 4) and D, E, and F (cf. Figure 5). Let us assume that vehicle A gets the green light and starts moving. The first vehicle needs to initialize the signal of departure for the others. We want the signal to be delivered only to vehicles standing behind vehicle A-in this example, vehicle B. Please see Figure 3, which presents a simplified situation at an intersection with two-lane roads. The simplification has been made in order to explain our idea in the most transparent manner. The shape of the wireless signal cannot be controlled, which is why it has been marked as a circle (representation of the range of signals emitted without any obstacles). Therefore, the authors proposed a suitable method for notification to be received by the appropriate vehicles. This is the reason why the desirable reaction area of the abovementioned signal has also been marked.

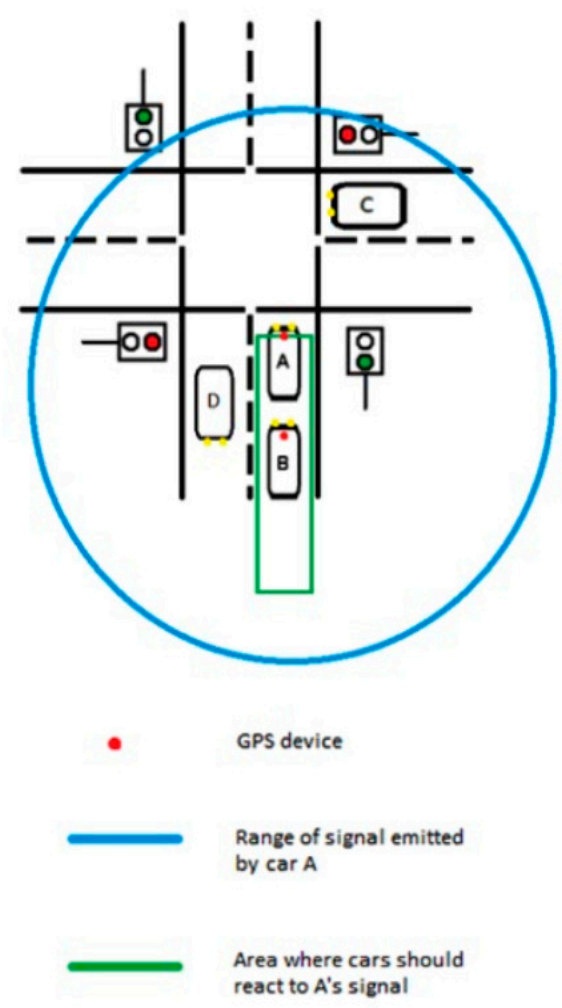

Figure 3. Top view of the simplified situation at the intersection. Range of signals emitted and desirable reaction area to abovementioned signal.

The idea that would solve the abovementioned problem is the vehicle's GPS tracking. The vehicle that is moving takes shots of its location at fixed intervals. After some time, it has a set of coordinates. We connect them by creating a trail, which is then sent to other vehicles. They can compare their current location with the received trail and tell if they are on the same road. Verhagen [37], with their augmentation system, are able to provide accuracy of a few centimeters [38]. The accuracy of several tens of centimeters is enough for the proposed method. The accuracy of geolocation depends upon a number of factors, e.g., the chosen signal (public or encrypted), the possibility of using more than one system of geolocation (single- versus dual-band solutions [39]) and surroundings: terrain, the number of visible satellites, reflections from tall buildings and the use of a regional or global localization system. The choice of global system, e.g., Galileo [39], also offers benefits in relation to the variety of available devices [40]. Considering the global trend, lower and lower prices make more and more precise GPS location systems available [27]. 
The proposed method can be used with ordinary smartphones on single carriageway roads. In such a case, no additional equipment is needed. Multi-lane intersections may be more challenging because precise location is crucial. Therefore, a modern smartphone with accurate satellite navigation is required.

The authors consider that, in the near future, most smartphones will be capable of precise location identification. The universality of such devices will allow us to apply the proposed method to multi-lane intersections without any additional equipment.

\subsection{A Method to Determine the Order of Vehicles at Traffic Lights}

A vehicle takes a location shot using a satellite navigation system. After about $1 \mathrm{~s}$, another location is taken and this creates a line segment with two points based on these locations. Then, another two line segments are created, which are parallel to the first and are the same length. In addition, their points lie on the line, which is orthogonal and passes through the first line segment points (see Figure 4).

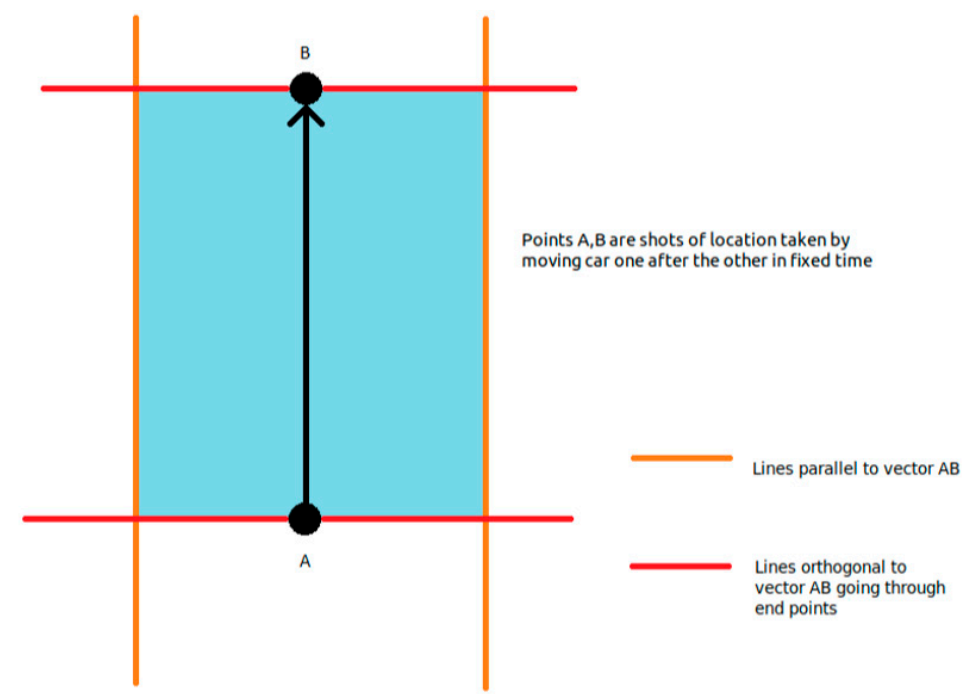

Figure 4. Rectangle generated by the algorithm to calculate the order of vehicles.

The vehicle should remember many such rectangles, which constitute the length of the trail. The relationship between shots and the number of remembered rectangles can be observed. Shorter intervals will make the trail more accurate in terms of position, but also shorter, if the same number of rectangles are to be kept in the system's memory. It is the choice of the implementer regarding the amount of available resources he or she has. In our simulations, we used a combination of applications: OMNeT++ [24], SUMO [23,41,42] and the Veins framework.

Objective Modular Network Tested in $\mathrm{C}++(\mathrm{OMNeT}++)$ is a written simulator in $\mathrm{C}++$ and also uses this language to describe simulated phenomena. The program can be used for the modeling and simulation of network protocols, the verification of hardware architecture, the assessment of performance aspects of complex software systems, network modeling communication, modeling queuing networks, etc.

The Simulation of Urban Mobility (SUMO) program is a powerful, microscopic motion simulator vehicle. SUMO allows for the configuration of vehicles, road networks and routes of cars and adapts them to the changing requirements of each project. Traffic Control Interface (TraCI) enables two-way communication with the simulator. This allows us to control the simulation process interactively and receive information about the current state of the objects in the simulation. By default, SUMO uses the Stefan Kraus model for the realistic modeling of each acceleration and deceleration of each vehicle [42].

The authors used the combination of $1 \mathrm{~s}$ and 20 rectangles, which seemed to be working correctly. This information is kept in the vehicle and needs to be sent to the other vehicles, which is where the VANET is used. 
For this purpose, the authors propose a special communication protocol. It is divided into four phases (please also see Figure 5-the diagram of the entire algorithm):

\subsubsection{Sending}

The first vehicle sends its ID, location and the coordinates (all four vertices) of the created rectangles. The ID must be a unique value; it can be the MAC address of the NIC (MAC) that is used for the communication.

\subsubsection{Receiving}

Other vehicles that are in the range of the signal receive the information. They check if their location is in the received area.

\subsubsection{Responding}

If so, they calculate the length between them and the sender and emit a signal with the sender's ID, its own ID and calculated length. The sender's ID is crucial because only by this data can the sending vehicle recognize that this answer applies to it.

\subsubsection{Matching}

At this point, the vehicle compares the lengths received from the previous point and selects the closest signal. As in the sending phase, the signal range cannot be controlled, so if a vehicle is answering, then all vehicles in the range receive the answer. They check if their ID is the same as that in the frame (as in the previous point described as "sender's ID"). After validation they compare the incoming length with that which is stored in the memory. If the incoming length is lower than in the memory, it is replaced along with an ID, which is now a pair. The default value stored in the memory is the positive infinity, so the first match will always be stored. After a few milliseconds, all answers should be considered, and the closest vehicle should be chosen.

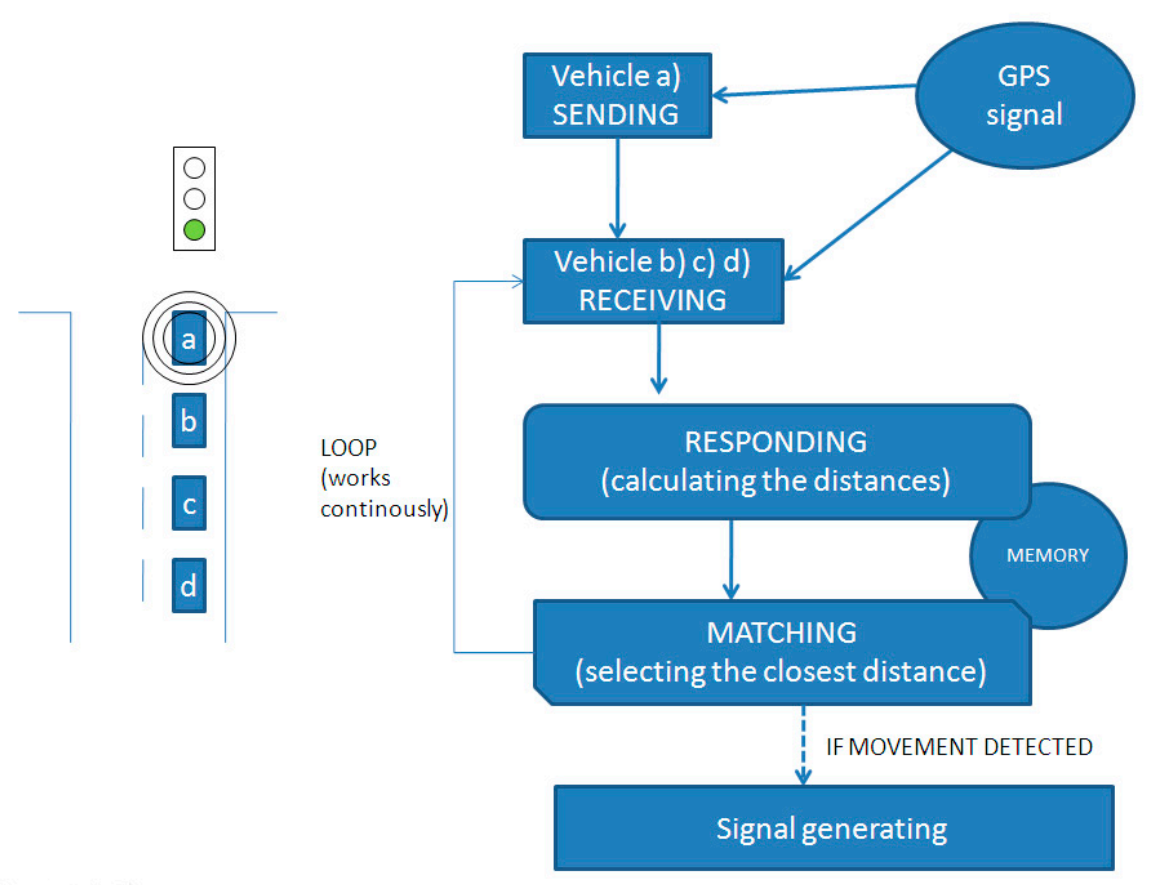

Figure 5. A diagram (visualization) of the described system architecture. 


\subsection{Example of Using the Methods Above}

Considering the situation shown in Figure 6, the vehicle designer decided to keep three rectangles in the memory, equaling 12 coordinates, while all vehicles are the same, i.e., each vehicle stores three rectangles. Rectangles stored in vehicle A's memory are depicted as grey and those in B are blue. The first few rectangles of vehicle A have been labeled in Figure 6: the first rectangle has four coordinates: A1, B1, C1, D1; the second rectangle's coordinates are A2, B2, C2, D2, and so on. All of these coordinates are calculated in the vehicle's CPU and emitted to all vehicles in range. Vehicle A stores three rectangles, and hence sends 12 coordinates.
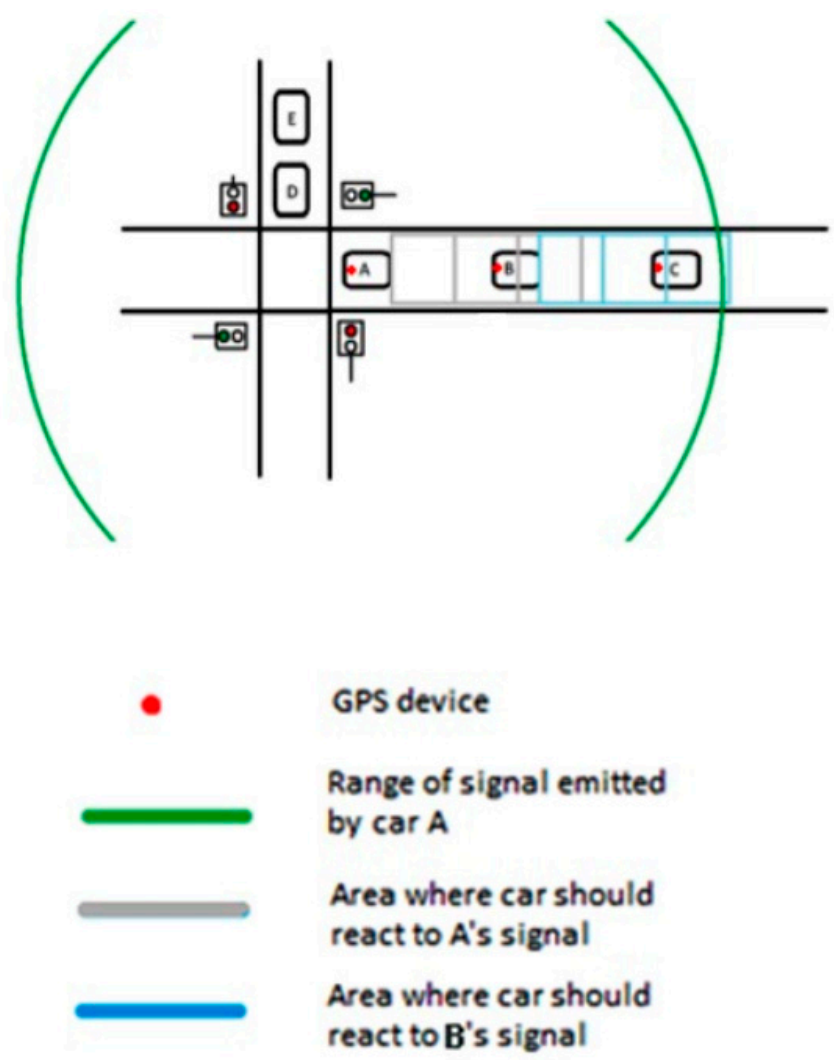

Figure 6. Algorithm working at intersection (simplified top view).

The sequence of events is as follows:

1. Vehicle A sends its ID, location and 12 coordinates (each coordinate consists of $x$ and $y$ );

2. Vehicles B, C, D, E, F receive A's information. B and C compare this with their location state to establish whether they are in the area;

3. At the same time, vehicle B also approaches the intersection and sends its own ID, location and 12 coordinates;

4. Vehicles A, C, D, E and F receive B's information. C and D state that they are in the area;

5. Vehicles B and C emit signals with their IDs, A's ID and the calculated length between them and A;

6. Vehicle A receives a response from $B$ first. It sees that it does not have a pair, so it creates a new pair. After a few picoseconds, from C's information, it compares the length with B, and it calculates that it is longer, then rejects it. Sending signals occurs in the domain of nanoseconds, so it can be a signal from $C$ that reaches vehicle A first. The order is caused by many factors and is hard to determine. The algorithm is therefore designed to be resistant to problems caused by signal "races".

7. Vehicle B receives a response from C and D. C happens to be closer, so it is chosen to create a new pair. 
The first vehicle does not belong to any area, which is important information, because only such a vehicle will be sending its initial signal to other vehicles, indicating its forward movement (setting off). There are two approaches to receiving a setting off notification-either all vehicles that trail the first vehicle receive the signal, or $B$ receives it from $A, C$ from $B$, and so on. In this project, the authors chose the first approach, as people can be alerted a few seconds earlier, before the vehicle in front of them moves off. Every vehicle receiving such a message sends its own duplicate, so the last vehicle in the first trail can be the first for subsequent vehicles. The question can be raised about the necessity of creating such a chain of vehicles, when the first approach is taken into consideration. The answer is that it is a good solution, because the first signal of the car that is moving will be sent only by the first vehicle in the chain, so there is no risk of interfering signals. Moreover, this is an excellent base for other projects because chaining is more difficult to implement and can be necessary in many situations. It is worth mentioning what happens if any vehicle does not implement VANET technology [43]. In this case, the vehicle without the system installed is treated as though it is not there. This technology informs the driver about the preceding vehicle moving forward only if a VANET system is installed. However, it is the driver's job to decide when to release the brake pedal and drive the vehicle. In other words, if the driver gets information and the non-VANET vehicle is still waiting in front, he or she may just use their horn. From the perspective of the system, this is not a problem, if the first and third vehicle have VANET and the second does not-in such a situation, the system sees two vehicles. This protocol requires transmitting a lot of data and many vehicles need to send these data simultaneously. The problem is that the transmitters can acquire only one transmission at a time, which is technically called the multithreading problem. In a wireless environment, it is impossible to manage the order of sending because of the necessity of sending data. The CSMA/CD (the full name of the protocol is: Carrier Sense Multiple Access with Collision Detection) protocol copes with it by establishing priorities-if the vehicle is unable to deliver a message, it attains a higher priority. In order to resolve this problem, the authors made efforts to limit the amount of data sent as much as possible. For example, the location signal (first phase) is sent only when the vehicle comes to a halt. Moreover, when it receives a pair, the interval of sending this signal is greatly extended. All transmissions are sent with some delay to minimize the chance of interfering signals [14].

It is worth mentioning that the functioning of the system is not directly based on traffic lights, but on the initial movement of vehicles when the preceding vehicle starts moving first. Using the algorithm, the leading vehicle may easily recognize the fact that it takes the first place in the queue simply because there are no messages coming in to it via VANET (there is no preceding car). When there is a need to stop, e.g., there is a fault with the traffic lights, the system will start to behave, i.e., to send and receive messages, in the same way as it does while waiting for a red light to change. Then, the following vehicles will start moving forward earlier, because the drivers supported by the sound signals react faster. Therefore, it can be stated that the system is robust in situations when a fault in traffic lights occurs, e.g., when lights start suddenly blinking.

\section{Results}

In this section, the authors discuss the results of two real-world experiments, which were based on measuring reaction times at an intersection. The authors used streaming and explored the possibility of shortening the reaction time of the driver through the sound signal information. Using the results from the abovementioned experiments, the authors configured road traffic simulations in a SUMO simulation that shows how much time may be saved using a solution based on a sound signal. The obtained results enable the authors to achieve (i.e., to observe) the headway between vehicles within an interval that is no less than $1 \mathrm{~s}$ [36].

\subsection{Reaction Time of Drivers to the Movement of the Preceding Vehicle}

In order to examine the reaction times of drivers at an intersection with traffic lights, a special website was created in the first half of 2019 [44]. The website was designed to carry out measurements 
with and without an audible warning of the vehicle in front moving away. The appearance of the website is shown in Figure 7. The entity responsible for the preparation of the website was the Scientific Circle CyberTech at the Faculty of Electronics of Wrocław University of Science and Technology.

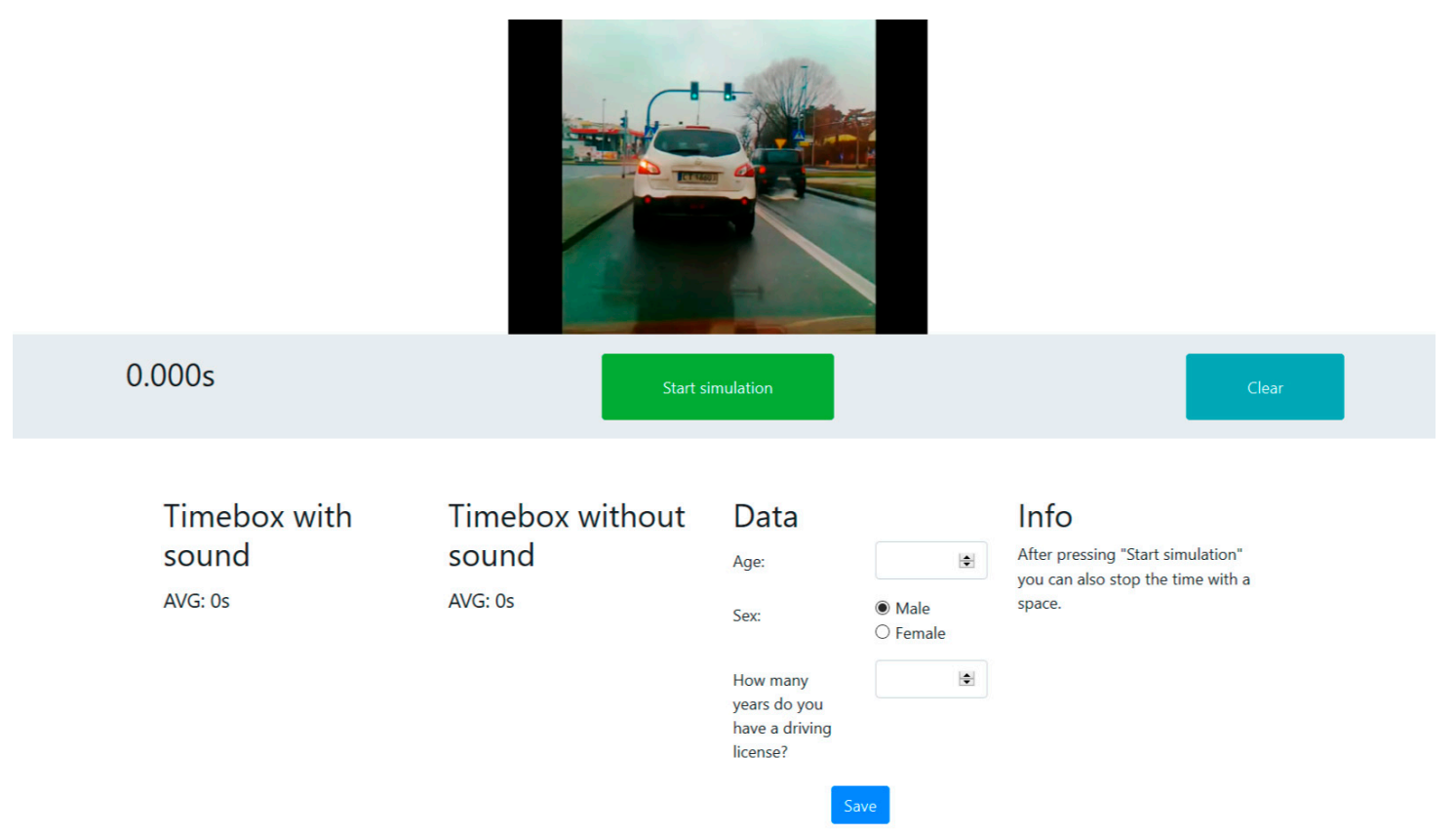

Figure 7. A fragment of the website created to measure the reaction time.

The process of using the abovementioned website is a very intuitive experience. Firstly, a tested person enters data about himself or herself, e.g., age, gender and how long he or she has had a driving license. These data were important to research in order to know if sound notification systems could be significantly more useful to drivers of certain age (e.g., for older individuals) or drivers with different levels of experience (e.g., for individuals with very little experience). However, none of these differences were observed. On the website, the reaction time measurements begin after the user presses the start button. The tested person is asked to press the stop button as soon as he or she notices that the preceding vehicle has started to move. The observations must be repeated. The minimum number of measurements was four for each person and the average number of measurements was 10,89. After completing the measurements, the user presses the save button to record the results [45].

In total, 161 individuals were examined, 150 men and 11 women, randomly selected from friends of the authors who use Facebook-using the convenience sampling method. The vast majority of those individuals were university students in the 18-24 age range (128 individuals and 1391 measured reaction times). In total, 24 individuals were in the $24-30$ age range (257 measured reaction times) and four individuals were older than 30 years old (50 measured reaction times). Five individuals did not reveal their age (55 measured reaction times). The majority of individuals were the holders of a driving license for a period of less than 6 years. Nobody was younger than 18 years old. The total number of measured reaction times was 1753, of which 1623 were men and 130 women.

Histograms of reaction times obtained with and without audible notification are presented in Figure 8 . Only five reaction times were longer than $2 \mathrm{~s}$; therefore, the axis of abscissas was scaled from 0 to $2 \mathrm{~s}$. 


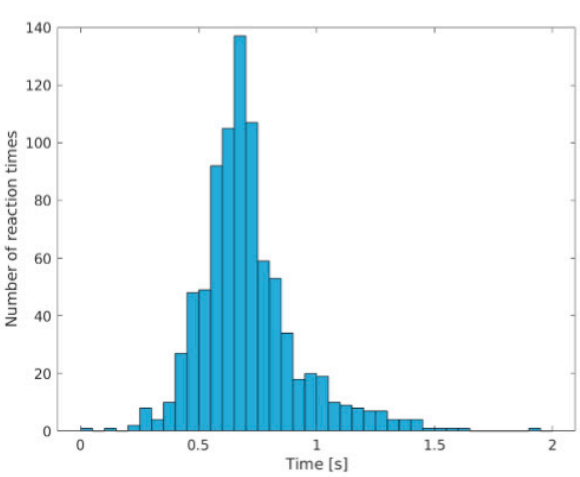

(a)

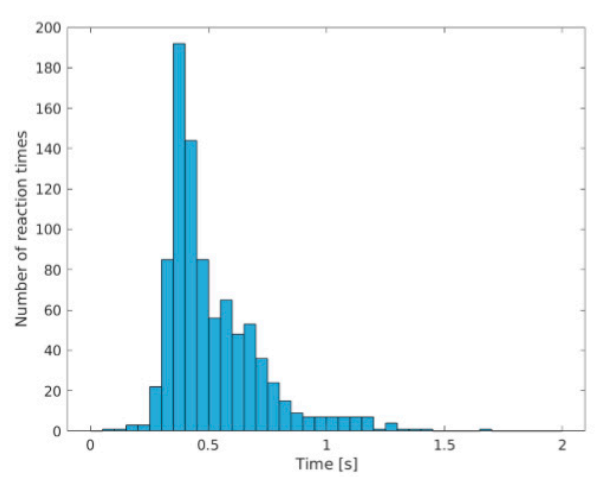

(b)

Figure 8. Reaction times. (a) Without sound; (b) with sound notification.

More statistics were calculated for reaction times and are depicted in Figure 8. Their values are presented in Table 1.

Table 1. Calculated statistics for measured reaction times.

\begin{tabular}{ccc}
\hline & Without Sound & With Sound Notification \\
\hline Median & $0.680 \mathrm{~s}$ & $0.446 \mathrm{~s}$ \\
First quartile & $0.585 \mathrm{~s}$ & $0.381 \mathrm{~s}$ \\
Third quartile & $0.797 \mathrm{~s}$ & $0.616 \mathrm{~s}$ \\
\hline
\end{tabular}

Based on the analysis of Figure 8 and data from Table 1, one can easily observe that the sound system has significantly shortened drivers' reaction times.

\subsection{Measuring Reaction Time Based on Video Recording}

\subsubsection{Measuring Reaction Time Based on Video Recording}

The recording from the monitoring camera at an intersection in Warsaw was used to measure the reaction time of drivers to the movement of the preceding vehicle. A view of the intersection is shown in Figure 9.

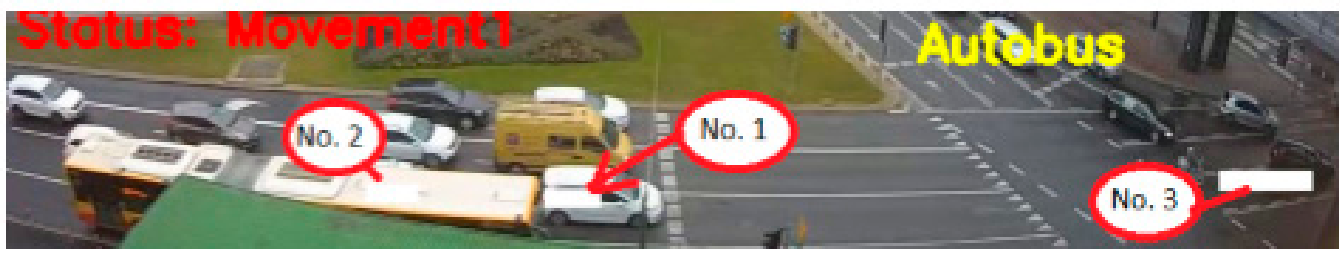

Figure 9. The intersection with marked places used for vehicle detection.

A video containing over 1000 cycles of light changes was recorded on a disk using a public camera. Based on an analysis of the way in which the filmed vehicles moved, 1034 response times were set out. In fact, the number of recorded cycles was probably slightly over 1034, because changes at lights where trucks or buses obstructed the movement of vehicles from places 1 and 2 (marked in Figure 9) had to be rejected. Measurements were also rejected when the third place was occupied and the car could not smoothly move from the lights.

For the automatic detection of vehicle movement and time measurement, a Python3 program was written using the OpenCV library, which automatically detected and determined response times. Figure 10 presents a fragment of the image processing sequence (digital image processing) that was used in the created program. In order to check the presence of vehicles in appropriate places, the absolute 
value of the difference between the current frame and the background is calculated. The background is changed to suit the time of day. To detect vehicle movement, the absolute value of the difference between the current frame and the previous one is calculated. Gaussian filters (with masks $5 \times 5$ and $21 \times 21$ ) are used to remove white noise from the image [46]. The transformation of the RGB image to a grayscale one is performed before or after Gaussian filtering. After the noise removal and transformation to grayscale, the image is treated as the threshold. This procedure is presented in Figure 10.

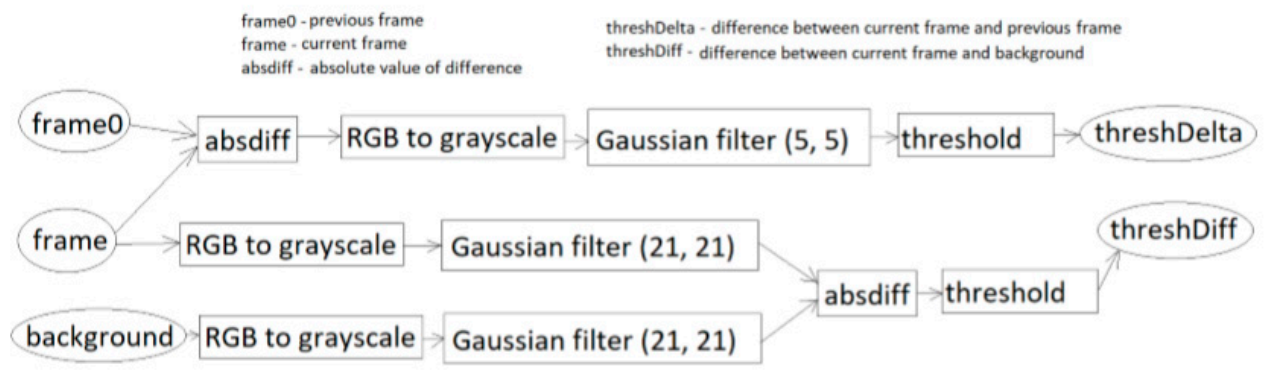

Figure 10. The use of Gaussian filters.

If vehicles are in places 1 and 2 by at least $2 \mathrm{~s}$, then the program waits for the movement of vehicle 1 . After the movement of vehicle 1, the program waits for the movement of vehicle 2 . When vehicle 2 moves, vehicle 1's reaction time is calculated. This time is treated as a single measurement. Additionally, if vehicle 1 moves in the situation of a traffic jam, then the measurement is rejected. A traffic jam is recognized as a situation where place 3 is occupied because the observed car cannot move smoothly in order to leave the intersection. Therefore, the authors find such a situation as not applicable to this research-the driver cannot react as fast they possibly can, even if he or she wanted to (please see Figure 9), so there is no point in measuring the time of his or her reaction. The locations and dimensions of places 1,2, and 3 are stored in a configuration file. It is worth mentioning that the previously described method of measuring reaction times is sensitive to some additional events, for instance, a bus that occupies two places at one time, coverage by vehicles from the next lane, etc. Therefore, the detection of long vehicles, such as buses or lorries, has been implemented in the program. Long vehicles are detected only in the vicinity of the lane where the measurement is performed. The detection of long vehicles is based on blob detection. A blob is defined as a group of connected pixels in image that share some common properties [5]. The blob detection criteria is the minimum area of the blob. This method is good for the detection of long vehicles, but one should not reject all incorrect measurements. Thus, after the program is executed, all video fragments between the movements of vehicle 1 and 2 are reviewed. This revision is supported by suitable Python script. The user may view fragments in sequence and accept or reject the video according to the appearance of some abnormalities. For example, one rejected fragment occurred due to a situation where a vehicle was occupying both lanes. This situation would have a distorting impact on the final measurements.

The number of obtained measurements is 1034 . The obtained reaction times are presented in Figure 9. The median is equal to $1.267 \mathrm{~s}$.

\subsubsection{Simulations of VANETs}

The performance of the new method for establishing the position of preceding vehicles is based a simple scenario of traffic lights standing alone on a single carriageway road. The simulation was implemented with 55 vehicles to observe the messages between traffic in the created VANETs. The authors focused their analysis on the following aspects:

1. the amount of time that is needed for the message from the predecessor to pass the information to the first, second, third, $\ldots, n$-th vehicle in the queue (where $n=55$ in simulations);

2. the number of messages that are broadcast from a single vehicle; 
3. the number of messages that are broadcast in total within a chosen period of time after a vehicle starts to move forward at an intersection;

All the messages sent between the 55 vehicles have been analyzed and are presented in Figure 11a-d. The data are received by a vehicle waiting in a queue; these data are transmitted by the preceding vehicle. Most of the time, just one message is generated, which causes an audio signal emission in the receiving vehicle (see Figure 11a). This transmitted message is confirmed by the successive vehicle. If a preceding vehicle receives a confirmation, there are no more messages exchanged between those two vehicles. However, occasionally, a successive vehicle will not confirm by sending a feedback response. This can happen due to various reasons (e.g., because it was processing a large number of other messages). Then, the successive vehicle has to repeat their message and the communication lasts longer, but such situations are very rare. Based on the empirical experience gained from the experiment, the authors consider that the abovementioned situation will not happen at this antenna signal range and the possibility of the distortion of data can be omitted.
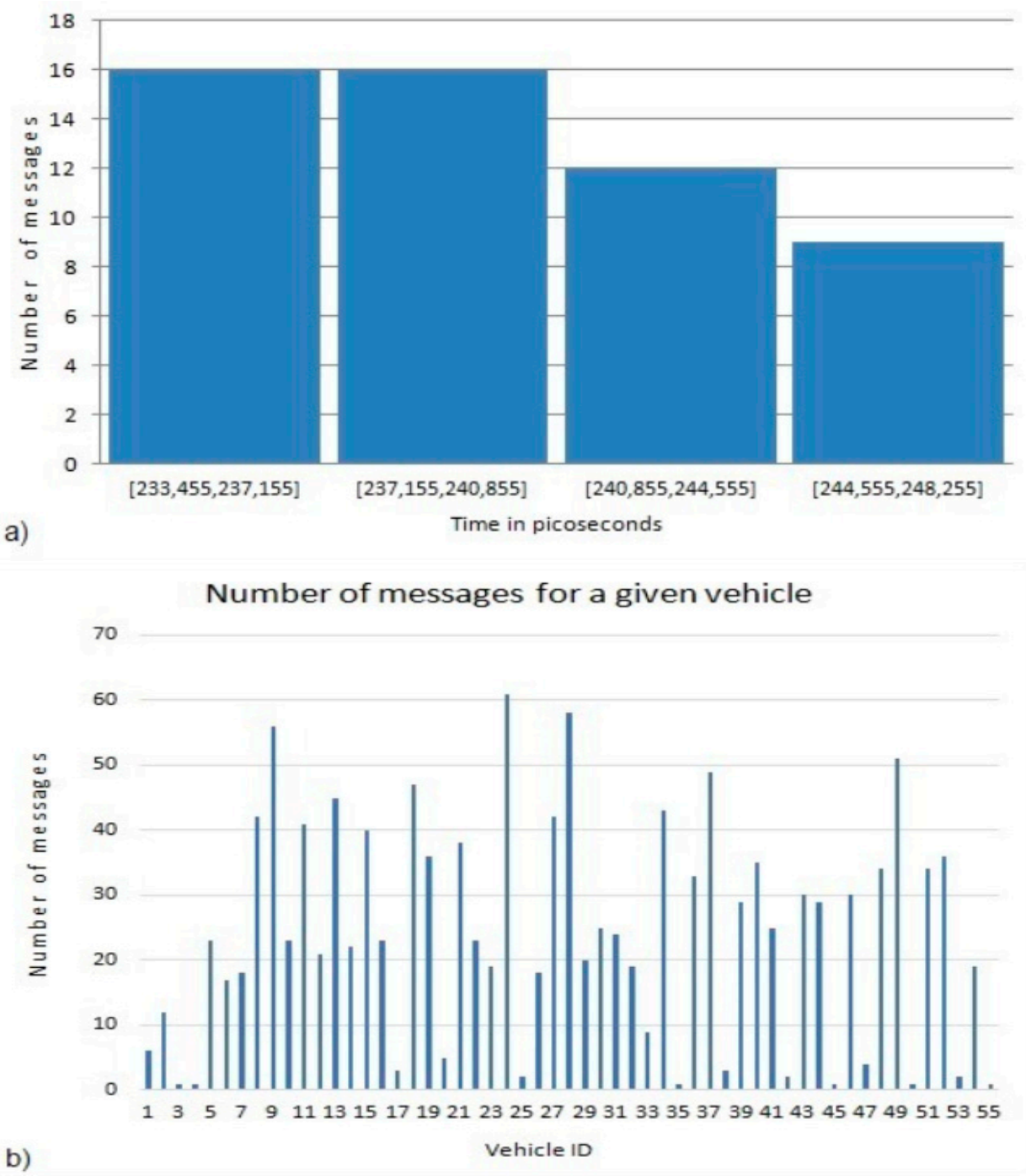

Figure 11. Cont. 
Number of messages before leaving the intersection

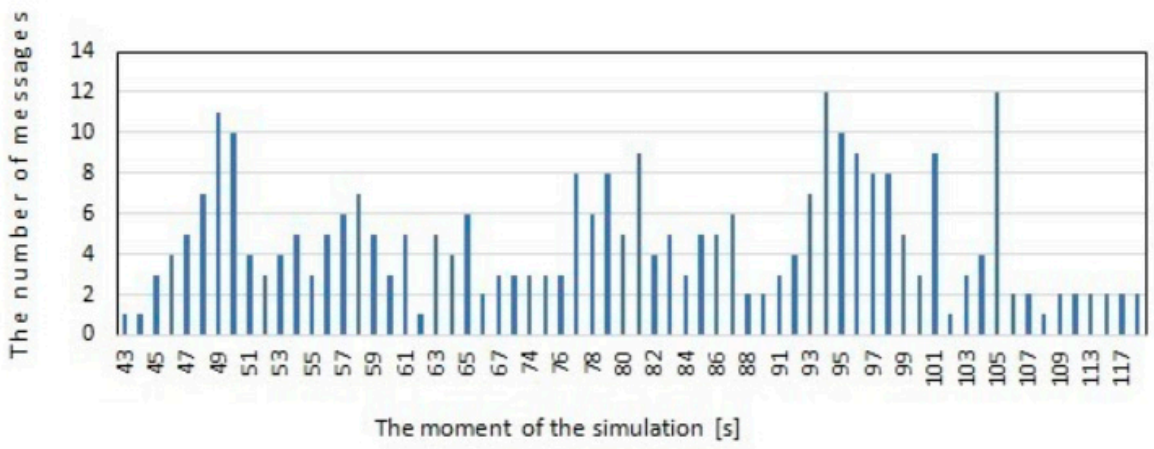

c)

Number of messages after leaving the intersection

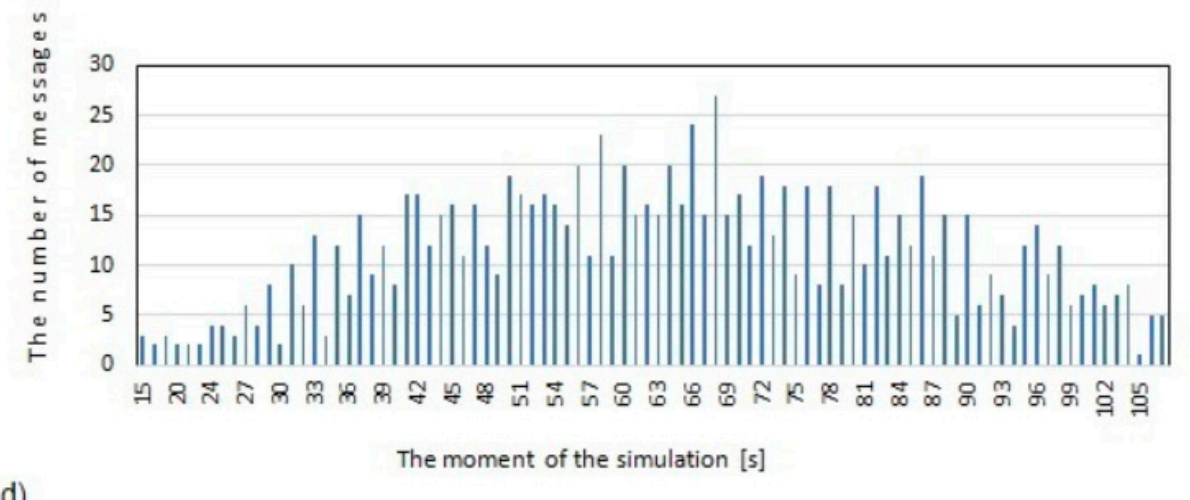

Figure 11. The network message flow at an intersection with one lane in one direction. The simulation contained 55 vehicles leaving the intersection after the change of the light from red to green.

Figure 11 presents the number of messages sent in subsequent moments after the movement of 55 vehicles in the OMNet simulator (the number of messages sent by all 55 vehicles in subsequent moments). Figure 11a shows the time needed for the message to reach the successive vehicles when sent from the preceding vehicle (these are only the messages that cause a sound signal in the receiving vehicle). Figure $11 \mathrm{~b}$ shows how many messages are broadcast from a single vehicle, Figure 11c shows the total number of messages broadcast within a chosen second of the simulation after the vehicles begin to move, but before they cross the intersection (junction). Figure 11d shows the total number of messages broadcast within a chosen second of the simulation after the vehicles begin to move when they have crossed the intersection.

\subsubsection{Simulations of Traffic Flow Improvements in SUMO}

The experiments were conducted using the open source simulator Simulation of Urban MObility (SUMO) [23,41,42]. A single carriageway road section with a length of $430 \mathrm{~m}$ was modeled, where a traffic light was placed $100 \mathrm{~m}$ before the end of the lane. The location of the traffic light was important. During the first $600 \mathrm{~s}$, the vehicles had time to line up in front of the lights and stop. Then (i.e., at time $t=600 \mathrm{~s}$ ), the light changes to green and the vehicles start moving. The simulation ended when the last vehicle left the intersection area. During the tests, the assumption was made that the reaction time of drivers had a probability density function that was specific to the skewed right distribution, because only a minority of drivers reacted to the change of lights with a considerable delay. A Python3 script was created, which allowed us to freely model the distribution of drivers' 
response times. The script reads the histograms of the distribution of tau $(\tau)$, as well as the distribution of the reaction time, i.e., action step length (ASL), from a file and then generates a result file with vehicle routing that is appropriate for the given distribution and saves it in the format accepted by the SUMO simulator. The SUMO simulator is based on the Krauss vehicle following model (cf. [23]), where, for any vehicle taking part in the simulation, the user assigns the values of the action step length (ASL) parameter and tau ( $\tau$ ) (cf. SUMO). The action step length parameter models the actual reaction time. In the simulation, there has to be a step length parameter that corresponds to the timesteps of the simulation, where any action of any driver is undertaken. The parameter tau $(\tau)$ corresponds to the headway in seconds in order to ensure the vehicle is at a safe distance from the preceding vehicle and the driver is able to react to the unexpected braking of the preceding vehicle. The $\tau$ parameter is dominant. This means that the total headway between vehicles, i.e., $\tau$, already includes the ASL time. Therefore, changing only ASL will not provide a significant change in the simulation time, if it is expected to model the shortening of the reaction time of the driver with respect to the changing traffic light from red to green (for the first driver) and the forward movement of the preceding vehicle (for the drivers in the subsequent vehicles). This is because every simulated vehicle's headway will remain unchanged. To model headway in SUMO, the parameter minGap also has to be set. minGap has a default equal to $2.5 \mathrm{~m}$; therefore, in the sound scenario, it is shortened proportionally with the shortening of $\tau$. Therefore, the effect of shortening the reaction time of the driver is modeled by shortening tau and setting $\operatorname{minGap}=2.5 \mathrm{~m} \cdot(\tau$ sound $/ \tau$ no sound) for each vehicle in the simulation. In the numerical experiments, the authors assume the shortening of $\tau$ by the difference between the median values measured by the web application (as mentioned above) from the previous section by $0.24 \mathrm{~s}$ (from $0.68 \mathrm{~s}$ to $0.44 \mathrm{~s}$ ). The tests were conducted based on the generation of 100 simulations according to the shifted distribution, for example:

1. for a simulation without sound notification, the ASL value is drawn randomly from the histogram obtained from the web application measurements without the buzzer (with the median $0.68 \mathrm{~s}$ )—see Figure $8 \mathrm{a}$ and Table 1 -then, $\tau$ is drawn randomly from the histogram obtained from camera measurements—see Figure 12 and $\operatorname{minGap}=2.5 \mathrm{~m}$;

2. for a simulation with sound notification, the ASL value is drawn randomly from the histogram obtained from the web application measurements with sound notification (with median $0.44 \mathrm{~s}$ ) — see Figure $8 \mathrm{~b}$ ) and Table 1 -then, $\tau$ is drawn randomly from the histogram obtained from camera measurements shifted to the left by $0.24 \mathrm{~s}=0.68 \mathrm{~s}-0.44 \mathrm{~s}$ and $\operatorname{minGap}=2.03 \mathrm{~m}$.

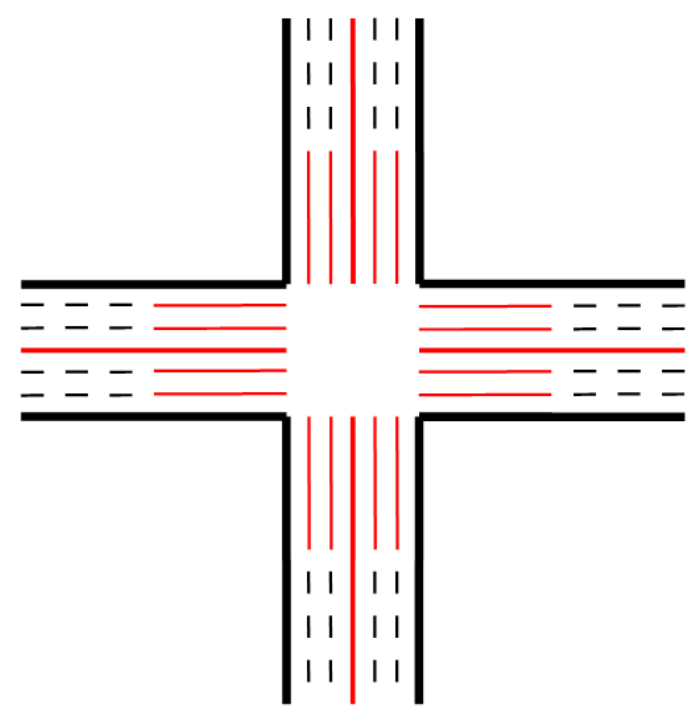

Figure 12. Visualization of intersection with multiple lanes (taken under consideration during work on algorithm of proposed solution). 
The time that the vehicle column needs to cross the intersection from the traffic lights was investigated using SUMO. The simulations were repeated 100 times, and then the results were averaged. The simulations were conducted on varying numbers of vehicles. Averaged times when the last vehicle in the column left the intersection are presented in Table 2. The second column in this table corresponds to the current state, that is, without a buzzer, i.e., headway at the expected level of $1.28 \mathrm{~s}$ and expected reaction time at the level of $0.68 \mathrm{~s}$ (Figure 8a). The third column corresponds to the speed up obtained by shortening the expected reaction time by $0.24 \mathrm{~s}$ from $0.68 \mathrm{~s}$ to $0.44 \mathrm{~s}$ (Figure $8 \mathrm{~b}$ ). At the same time, the expected headway changes to $1.04 \mathrm{~s}$. The fourth column corresponds to the simulated decrease in the headway to $0.9 \mathrm{~s}$, maintaining the reaction time at the expected level of $0.44 \mathrm{~s}$. From Table 2, it can be observed that shortening the reaction time by $0.24 \mathrm{~s}$, which is obtained from the measurements, significantly shortens the time needed to pass through the intersection. The time savings achieved are up to $13.5 \%$ (see Table 2 - third column). The fastest performance scenario for the algorithm based on sound signal notification and the shortening of drivers' reaction times was configured in the SUMO simulator, as close as possible to the results obtained in real-world measurements for a single carriageway road (as previously mentioned). In the conducted simulations, the reaction time distribution with and without a sound signal was set (see Figure 8), assuming an initial headway distribution corresponding to the camera measurement (Figure 9). In the SUMO simulation, it is assumed that the VANET communication time is negligible, and all vehicles have a functioning system, i.e., a GNSS device and smartphone application for sound notification.

Table 2 presents the times where the last vehicle in the column left the simulated area after the traffic lights changed. All data are presented as median values, i.e., the setup of ASL is drawn from measured distribution of the reaction time measured from the web application, $\tau$ is drawn from the measured distribution from the video (shortened by $0.24 \mathrm{~s}$ for the sound scenario) and minGap is set to the SUMO default value of $2.5 \mathrm{~m}$ and shortened proportionally with the shortening of $\tau$.

Table 2. The times where the last vehicle in the column left the simulated area after the traffic lights turned green.

\begin{tabular}{cccc}
\hline & Without a Sound Signal & \multicolumn{2}{c}{ With a Sound Signal } \\
\hline \multirow{2}{*}{ Number of vehicles } & ASL $=0.68 \mathrm{~s}$ & ASL $=0.44 \mathrm{~s}$ & ASL $=0.44 \mathrm{~s}$ \\
& $\tau=1.28 \mathrm{~s}$ & $\tau=1.04 \mathrm{~s}$ & $\tau=0.9 \mathrm{~s}$ \\
& $\operatorname{minGap}=2.5 \mathrm{~m}$ & $\operatorname{minGap}=2.03 \mathrm{~m}$ & $\operatorname{minGap}=1.75 \mathrm{~m}$ \\
\hline 25 & 55.78 & 48.01 & 44.15 \\
50 & 110.76 & 94.30 & 85.14 \\
75 & 170.24 & 146.49 & 134.29 \\
\hline
\end{tabular}

To reproduce the above experiment in a real-world scenario with the current level of geolocation standards, there a certain number vehicles must be equipped with external devices for GSNN, as described in Section 2.1. Such devices provide high precision location data about vehicles within lanes on a road. Moreover, each vehicle should be equipped with a smartphone with a proper application to calculate the order of the vehicles using the proposed algorithm (presented in Figure 5) and for communication between vehicles via the IEEE802.1 protocol. The abbreviation stands for Institute of Electrical and Electronics Engineers Standards Association for port-based Network Access Control protocol (also called shortly: PNAC). This part of the experiment corresponds with $\mathrm{OMNeT}++$ [24] simulations. As well as calculating the order of vehicles, the aim of the smartphone application is to generate a sound signal when the forward movement of the preceding vehicle is observed in order to shorten the reaction time of the driver. Therefore, the real-life reactions of drivers to the cars in front of them moving forward correspond to the SUMO simulation [23,41,42]. 


\section{Discussion}

The authors analyzed different situations of traffic flow in the context of described solution. The proposed method can be used at intersections both with and without traffic lights. After stopping the first car, the method causes automatic communication between vehicles in order to determine their order. It is worth mentioning that the proposed algorithm can be used not only at intersections, but also in other situations where cars stop for some time and then move again. Therefore, the proposed method can also improve traffic flow at level crossings. There are many intersections in urban areas, so it seems that improving traffic flow at intersections can be particularly beneficial. The algorithm will work best for the intersection of dual carriageway or single carriageway roads because, in this situation, it is easy to determine the order of the vehicles. An intersection with multiple lanes is shown in Figure 12. All red lines symbolize road markings that cars cannot cross. Thick red lines separate the opposite directions of vehicle traffic. Thin red lines symbolize the continuous lines that are often painted before intersections. The presented situation was taken into consideration when the algorithm of the solution was designed and, in the opinion of the authors, our algorithm should properly determine the order of the cars just before the intersection. However, the assumption has been made that drivers do not ignore traffic rules. It may be more problematic to properly chain cars in areas where lane changes are allowed. The trails driven by the vehicles are used to determine their order. The authors consider that it is very unlikely that a large number of cars in one lane will change traffic lanes at the exact same time in exactly the same place. Therefore, the algorithm will simply exclude the cars that change lanes from the computed chain of vehicles. In other words, the algorithm will treat those cars in the same way as vehicles without the system installed.

On the other hand, Figure 13 presents another possibility. A situation where, between vehicles using the proposed system (marked in green), there is a larger group of cars not equipped with the appropriate devices-these are figuratively marked in red. From the perspective of the proposed solution and inner algorithm, the presence of those red cars means that the first three vehicles will not be able to communicate with the last four green vehicles. In this situation, the proposed method will initiate the movement of the first green vehicles, and—after the red cars move-will then initiate the movement of the next four green vehicles.

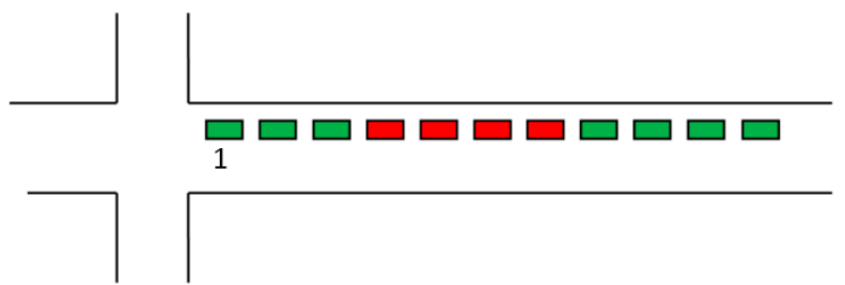

Figure 13. Four vehicles not equipped with proposed solution (in red) among series of cars with working sound notification system (in green).

Moreover, the measurement of moving cars at a real intersection was analyzed. Based on these studies, the distribution of reaction times was determined and is shown in Section 3.1. This allowed us to reasonably adjust the parameters of the simulation and model driving with and without the sound notification system. The authors are aware that, despite the way in which the research was conducted, the simulations may be somewhat different from real life traffic. For instance, differences in driving styles depend on cultural conditions. Furthermore, driving styles may vary from country to country. Therefore, Table 2 presents the simulation outcomes calculated for various parameter values. As a result, the impact of the proposed method can be more reliably assessed based on a comparison of the data from this table.

In terms of the existence of pedestrians, it must be said that, sometimes, they behave in an unexpected way, e.g., illegally crossing when the light is on green for vehicles. The authors would like to underline that the proposed solution is aimed at attracting the driver's attention to the road, 
i.e., drawing his attention away from (as already mentioned in this article) distractions. Even if a pedestrian steps into the road, the sound signaling informing about the moving vehicle ahead will attract the driver's attention to keep their focus on the road and he or she will see the threat. The authors would like to emphasize the fact that vehicles moving at an intersection have a very low speed, and hence that this is a safe solution and, above all, that it is strictly informative. The proposed system does not make any decisions itself, as it is not autonomous.

The authors would like to emphasize the assumptions taken under consideration during the development of the proposed solution (the observations of the authors confirm the following assumptions):

1. In the context of traffic light changes, the reaction time of each single driver can be shortened and the total amount time saved can be increased;

2. The reaction time of drivers has a probability density function, specific to the skewed right distribution, because only a minority of drivers react to the lights changing with a considerable delay;

3. In general, drivers do not ignore traffic rules.

The authors also consider that the limitations and constraints of the proposed solution must be highlighted. In the opinion of the authors, the main limitation of the described system is its strictly informative character-the system is not able to make any decisions and it is not capable of executing any decisions either. In addition, there is no component of artificial intelligence that could result in machine learning. On the other hand, in comparison to more developed intelligent transport systems, these limitations result in the extended control of the vehicle (by the driver) and an awareness of the situation on the road, which can positively influence safety. Moreover, our system, based on sound notification, is quite universal and will be useful even in the event of a small number of users-just two users (one after the other at a set of traffic lights) would be enough to make use of it.

\section{Conclusions}

The proposed solution, through the application of fundamental management functions and proper implementation, has the potential to reduce traffic jams and make traffic more fluid. This is confirmed by the simulation results shown in Section 3.2.3. In order to make the simulations correspond to reality, a dedicated website (presented in Section 3.1) was created and tests of drivers' reaction times to sound notifications were conducted. To the best of the knowledge of the authors, this is the first time that this type of test has been carried out.

The authors of this article indicate that the potential to reduce traffic jams and make traffic more fluid is a very important element.

Moreover, the solution does not require the construction of any new communication infrastructure. From a technical perspective, the developed algorithm for determining the order of vehicles is easy to implement. Its accuracy has been verified in the $\mathrm{OMNeT}++$ simulator, which allows for the testing of communication using various networks. Moreover, the authors of the article have made software that allows us to perform various future studies related to the analysis of the impact of reaction time on traffic flow at intersections. As shown in Section 4, even a small reduction in reaction time can significantly reduce the time needed to drive through the intersection. Based on the analysis of results from Section 4 , it can be assumed that, even if the proposed system only reduced the expected value of the reaction time by $0.24 \mathrm{~s}$, it still might reduce the time taken for the vehicle column to move by about $15 \%$ (when vehicles are fully synchronized) and by about $13.5 \%$ when reaction time is randomly chosen from empirical histograms. The rapid increase in the world population and the interrelated increase in the number of vehicles means that traffic on roads will continue to increase and the problem of traffic jams will become more and more critical. As mentioned in the introduction, traffic jams have many negative consequences. VANET networks are increasingly being used and it is probable the number of vehicles that can communicate using these networks will grow rapidly. 
It important to mention that a GPS receiver is necessary for the operation of the proposed method. However, this is not a significant problem, because the prices of GPS receivers are constantly decreasing as such equipment becomes more and more available. Furthermore, many currently manufactured vehicles are factory fitted with satellite navigation that includes a geolocation system or even more than one of this type of system. In addition to sound signal generation, other methods of signaling can also be used with the proposed method, such as visual information on a heads-up display placed on the windscreen, a method which is already used in many premium vehicles.

Finally, the authors of this paper state unequivocally that the aim of the paper has been achieved. Through the results of conducted research, it can be observed that the proper implementation of the proposed solution will certainly contribute to efficiency improvements for intelligent transport systems, while the proposed solution certainly has the potential to reduce traffic jams.

Author Contributions: Data curation, Ł.K.; Formal analysis, Ł.K., P.M., M.B., T.J., H.W. and T.C.; Funding acquisition, I.M.; Methodology, Ł.K., P.M., M.B., T.J., K.H. and T.C.; Project administration, T.J., H.W., I.M. and T.C.; Software, I.M., A.K., R.C. and J.S.; Supervision, M.B., T.J., K.H. and H.W.; Validation, M.B., T.J., K.H. and T.C.; Visualization, Ł.K., P.M. and T.C.; Writing-original draft, M.B., T.J., K.H., H.W., I.M., T.C., A.K. and R.C.; Writing-review \& editing, H.W. and T.C. All authors have read and agreed to the published version of the manuscript.

Funding: The project is financed within the framework of the program of the Minister of Science and Higher Education under the name "Regional Excellence Initiative" in the years 2019-2022; project number 001/RID/2018/19; the amount of financing PLN 10,684,000.00.

Acknowledgments: The authors would like to thank to Jakub Filinger-the member of the Scientific Circle CyberTech at the Faculty of Electronics of Wrocław University of Science and Technology who was responsible for writing our web application to measure reaction time with and without the audio signal.

Conflicts of Interest: The authors declare no conflict of interest.

\section{References}

1. Laurier, E. Notes on dividing the attention of a vehicle driver. Team Ethno Online, 2002, p. 1. Available online: http://www.teamethno-online.org.uk/Issue1/Laurier/gooddriv.html (accessed on 12 March 2020).

2. Shelton, J.; Kumar, G.P. Comparison between auditory and visual simple reaction times. Neurosci. Med. 2010, 1, 30-32. [CrossRef]

3. Jain, A.; Bansal, R.; Kumar, A.; Singh, K. A comparative study of visual and auditory reaction times on the basis of gender and physical activity levels of medical first year students. Int. J. Appl. Basic Med. Res. 2015, 5, 124. [CrossRef] [PubMed]

4. Kacker, S.; Saboo, N.; Sharma, M.; Nirvan, S. Effects of various distractions on audio and visual reaction time in 1st year MBBS students. Natl. J. Physiol. Pharm. Pharmacol. 2019, 9, 62-66. [CrossRef]

5. Choudhury, A.; Ramchandani, R.; Shamoon, M.; Khare, A.; Kaushik, K. An Efficient Algorithm for Detecting and Measure the Properties of Pothole. In Emerging Technology in Modelling and Graphics; Springer: Singapore, 2020; pp. 447-457.

6. Hadi, H.; Saleh, S.; Aljebori, T.H. A Survey on VANETs: Challenges and Solutions. Int. J. Eng. Technol. 2019. [CrossRef]

7. Hartenstein, H.; Laberteaux, K. Vanet: Vehicular Applications and Internetworking Technologies; Springer Science \& Business Media: Berlin, Germany, 2010; Volume 1.

8. Nellore, K.; Hancke, G.P.; Reindl Leonhard, M. A Survey on Urban Traffic Management System Using Wireless Sensor Networks. Sensors 2016, 16, 157. [CrossRef] [PubMed]

9. Wang, M.; Shan, H.; Lu, R.; Zhang, R.; Shen, X.; Bai, F. Real-time path planning based on hybrid-VANET-enhanced transportation system. IEEE Trans. Veh. Technol. 2014, 64, 1664-1678. [CrossRef]

10. El-Sersy, H.; El-Sayed, A. Survey of Traffic Congestion Detection Using VANET; Foundation of Computer Science FCS: New York, NY, USA, 2015; p. 1.

11. Nayak, R.R.; Sahana, S.; Bagalkot, A.S.; Soumya, M.; Roopa, J.; Govinda, R.; Ramavenkateswaran, N. Smart traffic congestion control using wireless Communication. Int. J. Adv. Res. Comput. Commun. Eng. 2013, $2,1-4$. 
12. Terroso-Saenz, F.; Valdes-Vela, M.; Sotomayor-Martinez, C.; Toledo-Moreo, R.; Gomez-Skarmeta, A.F. A cooperative approach to traffic congestion detection with complex event processing and VANET. IEEE Trans. Intell. Transp. Syst. 2012, 13, 914-929. [CrossRef]

13. Upadhyaya, A.N.; Chaturvedi, M.C. Traffic Congestion Detection and Avoidance using Vehicular Communication. Nirma Univ. J. Eng. Technol. 2015, 3, 1-7.

14. Al-qutwani, M.; Wang, X. Smart Traffic Lights over Vehicular Named Data Networking. Information 2019, 10, 83. [CrossRef]

15. Avzekar, M.K.; Moon, A. Adaptive traffic signal control with vanet. Int. J. Sci. Eng. Res. 2014, 2, $2347-3878$.

16. Kwatirayo, S.; Almhana, J.; Liu, Z. Adaptive traffic light control using VANET: A case study. In Proceedings of the 9th International Wireless Communications and Mobile Computing Conference (IWCMC), Sardinia, Italy, 1-5 July 2013; IEEE: Piscataway, NJ, USA, 2013; pp. 752-757.

17. Baras, S.; Saeed, I.; Tabaza, H.A.; Elhadef, M. VANETs-based intelligent transportation systems: An overview. In Advances in Computer Science and Ubiquitous Computing; Springer: Berlin, Germany, 2017; pp. 265-273.

18. Tomas-Gabarron, J.B.; Egea-Lopez, E.; Garcia-Haro, J. Vehicular trajectory optimization for cooperative collision avoidance at high speeds. IEEE Trans. Intell. Transp. Syst. 2013, 14, 1930-1941. [CrossRef]

19. Santamaria, A.; Tropea, M.; Fazio, P.; De Rango, F. Managing Emergency Situations in VANET Through Technologies Cooperation. Sensors 2018, 18, 1461. [CrossRef] [PubMed]

20. Chang, I.C.; Tai, H.T.; Yeh, F.H.; Hsieh, D.L.; Chang, S.H. A VANET-based A route planning algorithm for travelling time-and energy-efficient GPS navigation app. Int. J. Distrib. Sens. Netw. 2013, 9, 794521. [CrossRef]

21. Knorr, F.; Baselt, D.; Schreckenberg, M.; Mauve, M. Reducing traffic jams via VANETs. IEEE Trans. Veh. Technol. 2012, 61, 3490-3498. [CrossRef]

22. Hafeez, M.; Ahmad, R.; Hafeez, U. The future of vehicle crash avoidance through VANETs. Int. J. Adv. Appl. Sci. 2018, 5, 1-15. [CrossRef]

23. Krajzewicz, D. Traffic simulation with SUMO-simulation of urban mobility. In Fundamentals of Traffic Simulation; Springer: Berlin, Germany, 2010; pp. 269-293.

24. Fortino, G.; Russo, W.; Savaglio, C. Agent-oriented modeling and simulation of IoT networks. In Proceedings of the 2016 Federated Conference on Computer Science and Information Systems (FedCSIS), Gdańsk, Poland, 11-14 September 2016; IEEE: Piscataway, NJ, USA, 2016; pp. 1449-1452.

25. Marquis, B.L.; Huston, C.J. Leadership Roles and Management Functions in Nursing: Theory and Application; Lippincott Williams \& Wilkins: Philadelphia, PA, USA, 2009.

26. Sink, D.S. Productivity Management: Planning, Measurement and Evaluation, Control and Improvement; Wiley: New York, NY, USA, 1985.

27. Moore, S.K. Super-accurate GPS coming to smartphones in 2018 [News]. IEEE Spectrum. 2017, 54, 10-11. [CrossRef]

28. Moore, S.; Corner, J.; Haviland, J.; Wells, M.; Salmon, E.; Normand, C.; Brada, M.; Smith, I. Nurse led follow up and conventional medical follow up in management of patients with lung cancer: Randomised trial. BMJ 2002, 325, 1145. [CrossRef]

29. Wojtaszek, H.; Miciuła, I. Analysis of Factors Giving the Opportunity for Implementation of Innovations on the Example of Manufacturing Enterprises in the Silesian Province. Sustainability 2019, 11, 5850. [CrossRef]

30. Anthony, R.N.; Welsch, G.A.; Reece, J.S. Fundamentals of Management Accounting; RD Irwin: Washington, DC, USA, 1985.

31. Wojtaszek, H. A model system to motivate strategic element of innovative management in the production organization. Selected motivational aspects in the xyz manufacturing company. Case study. World Sci. News 2017, 72, 584-588.

32. Brancheau, J.C.; Wetherbe, J.C. Key issues in information systems management. MIS Q. 1987, 11, $23-45$. [CrossRef]

33. Hashiba, S.; Chang, T.C. Heuristic and simulated annealing approaches to PCB assembly setup reduction. In Human Aspects in Computer Integrated Manufacturing; Elsevier: Amsterdam, The Netherlands, 1992; pp. 769-777.

34. Szopik-Depczyńska, K. Effects of Innovation Activity in Industrial Enterprises in Eastern Poland. Oeconomia Copernic. 2015, 6, 53. [CrossRef] 
35. Lochert, C.; Scheuermann, B.; Mauve, M.; Hartenstein, H.; Laberteaux, K. Information Dissemination in VANETs. VANET Vehicular Applications and Inter-Networking Technologies; John Wiley \& Sons: Hoboken, NJ, USA, 2010; pp. 49-80.

36. Shinar, D. Reaction Time for Complex Stimuli, 2nd ed.; Emerald Publishing: Bingley, UK, 2017.

37. Verhagen, S. Performance analysis of GPS, Galileo and integrated GPS-Galileo. In Proceedings of the 15th International Technical Meeting of the Satellite Division of the Institute of Navigation (ION GPS-2002), Portland, OR, USA, 24-27 September 2002; pp. 2208-2215.

38. DoD, U. Global Positioning System Standard Positioning Service Performance Standard. Assistant Secretary of Defense for Command, Control, Communications, and Intelligence. 2001. Available online: https: //doi.org/10.1016/B978-0-444-89465-6.50070-3 (accessed on 12 March 2020).

39. Available online: https://www.gsa.europa.eu (accessed on 16 January 2020).

40. Karki, B.; Won, M. Characterizing Power Consumption of Dual-Frequency GNSS of a Smartphone. arXiv 2019, arXiv:1910.13041.

41. Behrisch, M.; Bieker, L.; Erdmann, J.; Krajzewicz, D. SUMO-simulation of urban mobility: An overview. In Proceedings of the SIMUL 2011, The Third International Conference on Advances in System Simulation, Barcelona, Spain, 23-29 October 2011.

42. Krajzewicz, D.; Erdmann, J.; Behrisch, M.; Bieker, L. Recent development and applications of SUMO-Simulation of Urban MObility. Int. J. Adv. Syst. Meas. 2012, 5, 128-138.

43. Jang, W.S.; Healy, W.M.; Skibniewski, M.J. Wireless sensor networks as part of a web-based building environmental monitoring system. Autom. Constr. 2008, 17, 729-736. [CrossRef]

44. Web Application to measure a driver's reaction time. Available online: http://driver-reaction.kubafilinger.pl (accessed on 13 June 2020).

45. Jha, A.R. Cryogenic Technology and Applications; Elsevier: Amsterdam, The Netherlands, 2011.

46. Rasheed, A.; Gillani, S.; Ajmal, S.; Qayyum, A. Vehicular ad hoc network (VANET): A survey, challenges, and applications. In Vehicular Ad-Hoc Networks for Smart Cities; Springer: Berlin, Germany, 2017; Volume 39-51.

(C) 2020 by the authors. Licensee MDPI, Basel, Switzerland. This article is an open access article distributed under the terms and conditions of the Creative Commons Attribution (CC BY) license (http://creativecommons.org/licenses/by/4.0/). 